\title{
Effectiveness of Human Detection from Aerial Images Taken from Different Heights
}

\author{
Muhammad Shahir Hakimy Salem, Fadhlan Hafizhelmi Kamaru Zaman, \\ Nooritawati Md Tahir
}

Faculty of Electrical Engineering, Universiti Teknologi Mara, 40450 Shah Alam, Selangor, Malaysia

\begin{abstract}
Recently, drones have been regularly used to aid in search and rescue in places where it is normally to carry out some of the early forensic victim localization. There are many suitable human detectors for drone use, such as Histogram Oriented Gradient (HOG), You Only Looks Once (YOLO), and Aggregate Channel Features (ACF). In this paper, the height of the aerial images is analyzed for its effect on the accuracy of the detection. This works compares ACF, YOLO MobileNet, and YOLO ResNet50 using a different set of aerial images varying at $10 \mathrm{~m}, 20 \mathrm{~m}$, and $30 \mathrm{~m}$ heights. The results show that in a single-model test, with our proposed bounding-box standardization, YOLO MobileNet achieves significant increase in test precision (AP), with 0.7 AP recorded. For single-model test, YOLO MobileNet yield best AP using $20 \mathrm{~m}$ training data where it obtained AP of 0.88 (10m test height), 0.82 (20m test height), and 0.91 (30m test height).
\end{abstract}

Keywords - Human Detection, Aerial Images, YOLO MobileNet, YOLO ResNet50, ACF.

DOI: 10.18421/TEM102-06

https://doi.org/10.18421/TEM102-06

Corresponding author: Fadhlan Hafizhelmi Kamaru

Zaman,

Faculty of Electrical Engineering, Universiti Teknologi Mara, 40450 Shah Alam, Selangor, Malaysia

Email: fadhlan@uitm.edu.my

Received: 31 October 2020.

Revised: 02 March 2021.

Accepted: 09 March 2021.

Published: 27 May 2021.

(c))BY-NC-ND (C) 2021 Muhammad Shahir Hakimy Salem, Fadhlan Hafizhelmi Kamaru Zaman, Nooritawati Md Tahir; published by UIKTEN. This work is licensed under the Creative Commons Attribution-NonCommercial-NoDerivs 4.0 License.

The article is published with Open Access at www.temjournal.com

\section{Introduction}

In recent years, there has been a rapid increase in the use of Unmanned Aerial Vehicles (UAVs) in various applications that include search and rescue (SAR) operations [1], disaster management [2], and many more areas that specifically require information about the detection of human beings [2]. Further, in several applications, it is indeed essentially for precise human detection to be conducted especially at a different height. In addition, shadows, uncontrolled lighting environments, and shaky videos can all add up to the difficulty of this task.

Moreover, the task of human detection in aerial image sequences is challenging due to various reasons. One of the main reasons that contributed to this factor is the variation of human size in the video frame due to the location or height of the video capture used during image acquisition [3]. The higher the video location from the human subject, the smaller the size of the human image in the video frame is. Hence height is one of the limitations in the aerial image in human detection and tracking process. Also, the accuracy of human detection and rapid training time are the two important keys that should be taken into consideration for a robust human and non-human classification system [3].

Therefore, in this study, three different heights of aerial images are investigated to verify that differences in height is one of the factors that could decrease the detector performance. Additionally, besides height as one of the issues, other factors could also affect the detector performance such as lighting, camera angle, and the human themselves. However, in this work, only height will be considered and investigated. The rest of this paper is arranged as follows. Section 2 outlines some related work and applications that utilized aerial images. Section 3 describes and elaborates details of the methodology and specification of the dataset that is used in this study. Section 4 analyses and discusses the results obtained from the experiments conducted using each detector namely ACF, YOLO MobileNet, and YOLO ResNet50. Finally, Section 5 is the conclusion and proposed future work. 


\section{Literature Review}

This section discussed the overview method of aerial image human detection and tracking followed by the evolution of aerial image in human detection algorithms toward a deep-learning approach. For the past decades, human detection and tracking studies has been an active research field, which have been evolving and made good progress in different applications and scenarios. As mentioned earlier, there are several types of input images that can be used in human detection and tracking and one of them is an aerial image. Note that an aerial image is an image that is acquired or captured from an aircraft or other flying objects [4] such as drones. Aerial images are excellent in capturing textured regions with distinct signatures, for instance areas such as water, land, vegetation, and so forth. There are many applications using aerial images such as disaster relief, urban planning, and real estate management.

Aerial images intended to be used in human detection and tracking need to overcome several issues including image quality, resolution, brightness, and angle. Another challenging factor is the variation of human size in the video frame [3] due to the dissimilar altitude of the camera platform during the image acquisition task [3]. It is not possible to combat all the factors mentioned here, but recently with the deep learning (DL) algorithm, it becomes an easier task. DL is proven as one of the most popular approaches as there are much research involving deep learning in human detection and tracking as reported in [1], [3], [5], [7]. The two main steps in human detection and tracking are feature extraction and detection. Some methods or techniques that are already established are discussed below.

\subsection{Feature Extraction}

The process of feature design and selection is so sensitive because it has a significant role in improving the performance of the detector. Various factors affect the performance of the detector and one of the factors is diversity of the datasets. Feature learning methods are fruitful for large datasets with thousands of classes due to domain adaptation [3]. These datasets are robust against the dynamical events in different scenarios. One of the supervised features learning method is the convolution neural network $(\mathrm{CNN})$. There are many feature extractions examples that can extract an import feature from the aerial images such as Scale Invariant Feature Transform (SIFT) [5], Local Binary Pattern (LBP) [6] and Histogram oriented gradient (HOG) [7]. HOG preserves information concerning the gradient surrounding detected spatial interest points and it was proven to be robust against changes in human appearance [7].

\subsection{You Only Looks Once (YOLO)}

Detection is a process of determining the location on the image where certain objects are presently followed by classifying those objects. There are many types of deep-learning architecture namely convolutional neural network $(\mathrm{CNN})$, recurrent neural network (RNN), generative adversarial neural network (GAN), ResNet and others. Each type of deep-learning architecture mitigates a different type of problem. CNN has been established as one of the most promising avenues of research in the computer vision area, providing outstanding results in a series of visual recognition tasks, such as image classification [8], [9], face recognition [10], digit recognition [11], [12], pose estimation [13], object and pedestrian detection [14], [15], and contentbased image retrieval [16], [17]. CNN consist of multilayer perceptron neural networks. A general CNN architecture is made of a few numbers of convolutional layers, pooling layers, a fully connected layer, and a normalization layer. This is inspired by the biological process of organizing the animal visual cortex [18]. It has also been demonstrated that features extracted from the activation of a CNN trained in a fully supervised fashion on a large, fixed set of object recognition tasks can be repurposed to novel generic recognition tasks [19].

Additionally, deep learning can also be used as a detection algorithm. There are various detection models in the detection process using a deep-learning approach. The most popular and current excellent target detection models are R-CNN [14], SPP-Net [20], Fast-RCNN [21], Faster-RCNN [22], SSD [23], YOLO [24], and ResNet [25]. Amongst these, YOLO is the fastest object detector with similar accuracy as R-CNN [26]. YOLO performs CNN-based object recognition in a box called an anchor and will be used during the training and detection process. The training images should be pre-processed to determine satisfactory accuracy using YOLO [26]. The size of the training images and detection images should be the same. The proportion of the area occupied by the object in the images has to be similar in the training images and the detection imaged. With the recent version of YOLO known as YOLOv2 and YOLOv3, training and performance can be increased based on this latest version.

\section{Methodology}

The methodology adopted in this work can be described as follows. It can be divided into four different parts as shown in Figure 1. 


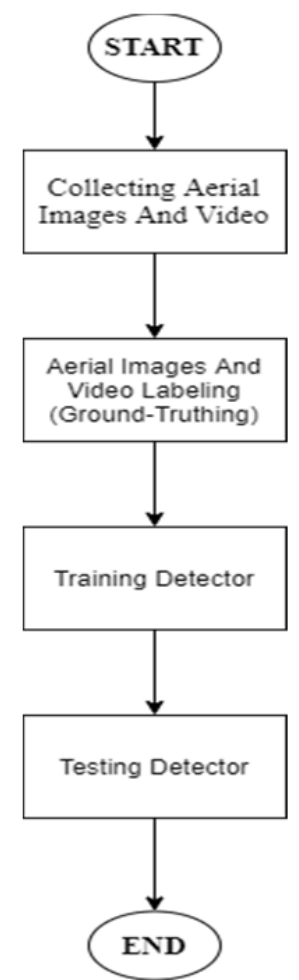

Figure 1. Overview of the Proposed Methodology

\subsection{Aerial Images and Videos Acquisition}

Firstly, the database of the aerial image which includes targets and background images is acquired. Note that the target aerial images may consist of partial human body parts like head, face, hand, foot, finger, and some other parts that could represent a human body. In addition, the images are taken at different heights as well. Meanwhile, the non-target image or the background image should also include some other objects to represent non-human objects. For database development, three different types of height that will be evaluated are taken at 10 meters, 20 meters, and 30 meters, respectively using a Phantom 3 Professional drone with $4 \mathrm{~K}$ resolution camera. The maximum height is set at 30 meters since it is almost impossible to perform the target labelling process for 30 meters and above. Every trained detector will be tested against these three different heights of datasets to determine its recall and precision rate where MATLAB is used for the ground truth labelling. Tables 1 and 2 tabulated the data collection and specification of the database for this study.

Table 1. Information on Data Collection

\begin{tabular}{|c|l|}
\hline Location & Dataran Kemerdekaan Shah Alam \\
\hline Period & $\begin{array}{l}\text { 16 December 2019 Until 18 } \\
\text { December 2019 }\end{array}$ \\
\hline Equipment & $\begin{array}{l}\text { DJI Phantom 3 Professional } \\
\text { Quadcopter }\end{array}$ \\
\hline Coordinate & $3^{\circ} 04^{\prime} 36.6^{\prime \prime} \mathrm{N} 101^{\circ} 31^{\prime} 24.4^{\prime \prime} \mathrm{E}$ \\
\hline
\end{tabular}

Table 2. Specification of Database

\begin{tabular}{|l|c|}
\hline Type of Videos & 3 \\
\hline Duration of Videos & 1 Minute \\
\hline Size of Videos & $400 \mathrm{Mb}-600 \mathrm{Mb}$ \\
\hline Heights & 10 Meter, 20 Meter, 30 Meter \\
\hline Image Resolution & $4096 \times 2160 \mathrm{p}(4 \mathrm{~K})$ \\
\hline
\end{tabular}

Further, Figure 2 depicted the three setting or situation during image acquisition that is captured at three different height. It is clearly seen that the size of the human form indeed varies due to the dissimilar heights.

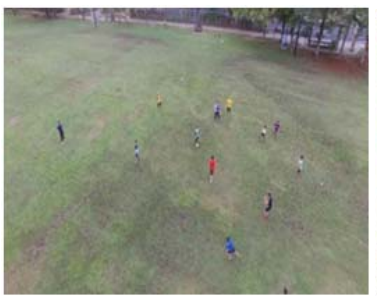

(a)

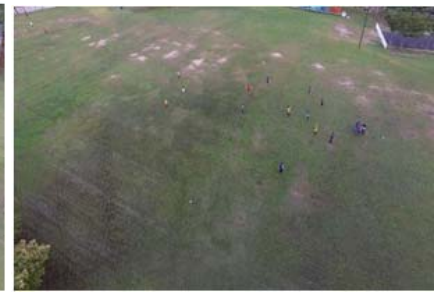

(b)

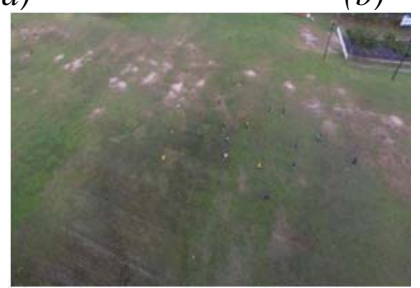

(c)

Figure 2. Database Acquisition at three different heights: (a) 10 meter (b) 20 meter and (c) 30 meter

\subsection{Aerial Images and Videos Ground Truth Labeling}

Image and video labeling are vital prior to detector training. Upon completion of aerial images and video acquisition, next is object labeling in both images and videos by specifying a bounding box around each object, and here the object that has to be labeled is a human form. The ground truth labeling for both aerial images and video labeling process is done using Matlab.

As for the labeling process, there are four types of algorithms that can be chosen for a detector. In this case, the ACF People Detector is selected. Furthermore, to ensure varieties in the ROI, every few frames are excluded based on changes in ROI. The images are extracted in between 5 to 15 seconds and then omitted for 10 seconds, and this process continued to Figure 3 that shows the ground-truth process for each dataset. Every dataset is developed based on the ground truthing process with each image of a human or subject in the ROI are labeled. 
Table 3. The collected aerial data

\begin{tabular}{|c|c|c|c|}
\hline $\begin{array}{c}\text { Drone } \\
\text { Height } \\
\text { (Meter) }\end{array}$ & $\begin{array}{c}\text { Human } \\
\text { Size } \\
\text { per } \\
\text { Frame }\end{array}$ & $\begin{array}{c}\text { Number of } \\
\text { Frames } \\
\text { for each } \\
\text { Video }\end{array}$ & $\begin{array}{c}\text { Total human } \\
\text { labeled }\end{array}$ \\
\hline $\mathbf{1 0}$ & Large & 195 & 2730 \\
\hline $\mathbf{2 0}$ & Medium & 195 & 2730 \\
\hline $\mathbf{3 0}$ & Small & 146 & 2044 \\
\hline
\end{tabular}

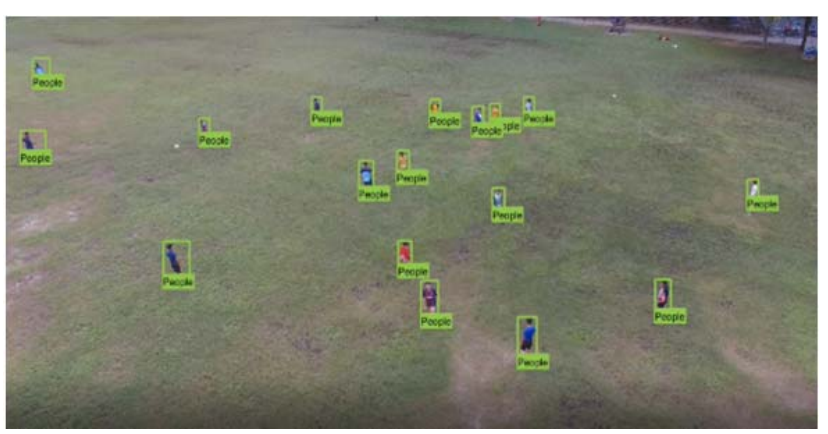

(a)

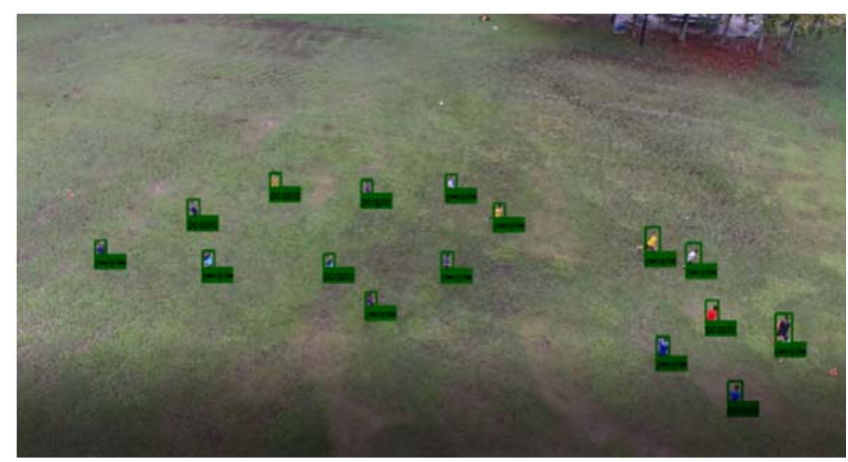

(b)

Figure 3. Samples of images at location (a) 10-meter (b) 20-meter datasets based on ground truth labeling

\subsection{Training the Detectors}

This training part is the most important part. Three types of detectors are utilized in this study namely ACF, YOLO MobileNet, and YOLO ResNet50 detector. Next, every detector is trained using three different heights $(10 \mathrm{~m}, 20 \mathrm{~m}, 30 \mathrm{~m})$ of the dataset that has been labelled during the ground-truthing process. Next, every detector will be evaluated and validated against three different datasets $(10 \mathrm{~m}, 20 \mathrm{~m}, 30 \mathrm{~m})$ based on the database from entirely different sessions. Hence, every detector is trained and tested using datasets acquired at different sessions.

\subsubsection{ACF Detector}

ACF detector consists of four stages of the training process that include sample negative example, compute approximation coefficients, and aggregated channel features. Every stage will train the classifier with positive and negative examples. For every stage, the weak learner will appear. The total dataset used for the ACF detector is 149 images and each image comprised of 14 images of human that have been labeled accordingly. In total there, 2730 images are labeled as humans act as databases for the training phase.

\subsubsection{YOLO Detector}

Next is the YOLO detector. For the YOLO detector, the suitable number of anchor boxes needs to be computed prior to modeling. In this study, every trained detector needs to find suitable anchor boxes as optimal IOU (Intersection Over Union) as shown in Figure 4. Figure 4 shows that the optimum anchor box is 4 for 10 -meter training detector model. A suitable number of anchor boxes will affect the training process of the detector. Figure 4 shows the results horizontal from 4 to 15 numbers of anchors. The higher the number of anchor box, the longer the time taken for the training process.

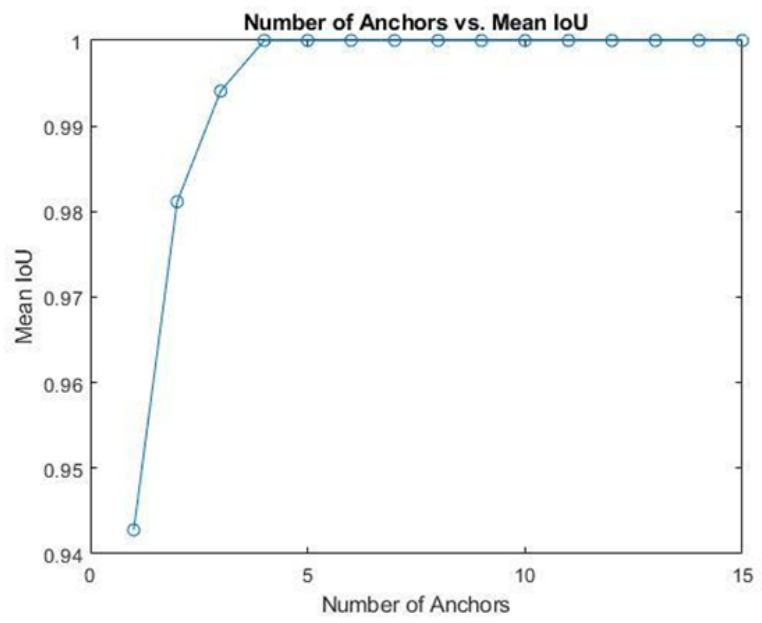

Figure 4. Examples result of finding a suitable number of anchor boxes for 10-meter detector

Further, the YOLO detector is evaluated and validated using MobileNetV2 and ResNet50 architecture as a pre-trained network. Note that Mobilenet and ResNet50 are the Convolutional Neural Network (CNN) architecture model for image classification and mobile vision. MobileNet architecture owns small and low latency. Figure 5 shows the parameter of MobileNet architecture. For MobileNet, the total number of layers is 28 considering depth wise and point wise convolution as separate layers.

However, with MobileNetV2 two new features to the architecture that is linear bottlenecks between the layers [27] and shortcut connections between the bottlenecks [28] are introduced and added. Hence, MobilenetV2 models are faster for some accuracy across the entire latency spectrum. This MobileNetV2 still uses the depth wise separable convolutions as efficient building blocks. Figure 6 depicts the main building block for MobileNetV2. 


\begin{tabular}{|c|c|c|}
\hline Type / Stride & Filter Shape & Input Size \\
\hline Conv / s2 & $3 \times 3 \times 3 \times 32$ & $224 \times 224 \times 3$ \\
\hline Conv dw / s1 & $3 \times 3 \times 32 d w$ & $112 \times 112 \times 32$ \\
\hline Conv/s1 & $1 \times 1 \times 32 \times 64$ & $112 \times 112 \times 32$ \\
\hline Conv dw / s2 & $3 \times 3 \times 64 d w$ & $112 \times 112 \times 64$ \\
\hline Conv/s1 & $1 \times 1 \times 64 \times 128$ & $56 \times 56 \times 64$ \\
\hline Conv dw / s1 & $3 \times 3 \times 128 \mathrm{dw}$ & $56 \times 56 \times 128$ \\
\hline Conv/s1 & $1 \times 1 \times 128 \times 128$ & $56 \times 56 \times 128$ \\
\hline Conv dw / s2 & $3 \times 3 \times 128 d w$ & $56 \times 56 \times 128$ \\
\hline Conv / s1 & $1 \times 1 \times 128 \times 256$ & $28 \times 28 \times 128$ \\
\hline Conv dw / s1 & $3 \times 3 \times 256 \mathrm{dw}$ & $28 \times 28 \times 256$ \\
\hline Conv/s1 & $1 \times 1 \times 256 \times 256$ & $28 \times 28 \times 256$ \\
\hline Conv dw / s2 & $3 \times 3 \times 256 d w$ & $28 \times 28 \times 256$ \\
\hline Conv/s1 & $1 \times 1 \times 256 \times 512$ & $14 \times 14 \times 256$ \\
\hline $5 \mathrm{x}$ Conv dw / s1 & $3 \times 3 \times 512 d w$ & $14 \times 14 \times 512$ \\
\hline Conv / s1 & $1 \times 1 \times 512 \times 512$ & $14 \times 14 \times 512$ \\
\hline Conv dw / s2 & $3 \times 3 \times 512 d w$ & $14 \times 14 \times 512$ \\
\hline Conv/s1 & $1 \times 1 \times 512 \times 1024$ & $7 \times 7 \times 512$ \\
\hline Conv dw / s2 & $3 \times 3 \times 1024 d w$ & $7 \times 7 \times 1024$ \\
\hline Conv/s1 & $1 \times 1 \times 1024 \times 1024$ & $7 \times 7 \times 1024$ \\
\hline Avg Pool/s1 & Pool $7 \times 7$ & $7 \times 7 \times 1024$ \\
\hline $\mathrm{FC} / \mathrm{s} 1$ & $1024 \times 1000$ & $1 \times 1 \times 1024$ \\
\hline SoftMax / s1 & Classifier & $1 \times 1 \times 1000$ \\
\hline
\end{tabular}

Next, Figure 7 shows the overall flow chart for the training process of the YOLO detector. The database is split into training and testing database. Next, the input images are resized to $448 \times 448$ pixels. As for YOLO, two types of pre-trained network that is MobileNet and ResNet50 has been used. The convolution layer filter size is $3 \times 3$ that is common in CNN architectures. Experimental analysis using YOLO consists of six detectors that is YOLO MobileNet detector at three different heights at $10 \mathrm{~m}$, $20 \mathrm{~m}$, and $30 \mathrm{~m}$ detector and YOLO Resnet50 as well. All the detectors will further be used for testing using unseen databases specifically different datasets which are used as training datasets.
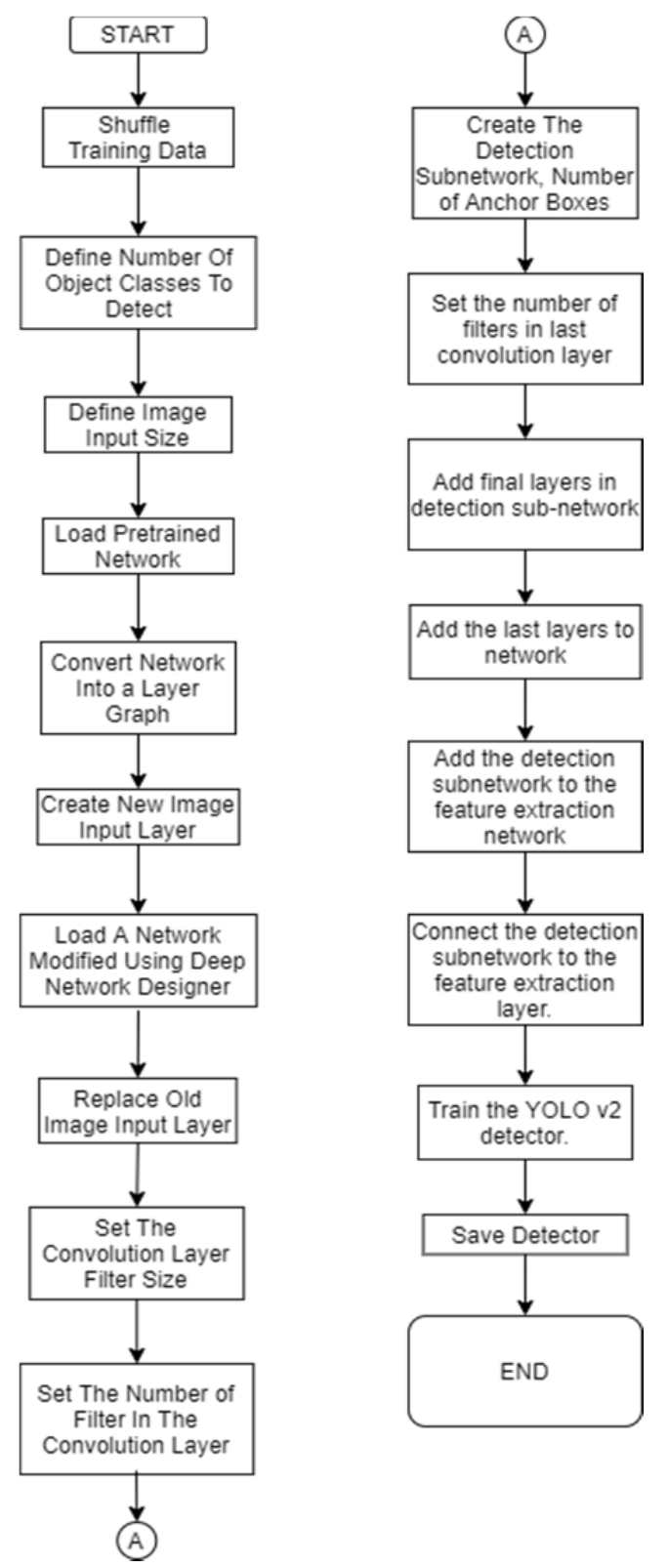

For a more effective detector training process, the training data has been shuffled prior to the training process. The training parameters that are used in this experiment are mini-batch size $=30$ and maximum

Figure 7. Flow chart on the training process of the YOLO detector 


\section{Result and Discussion}

In this experiment, 3 types of detectors are used, namely ACF, YOLO MobileNet, and YOLO ResNet50. Preliminary results showed that the original ground truths, which were too tight, badly affects the accuracy of the detection. The original bounding box causes over fitting, and the trained detectors failed to generalize well on the test dataset. The bounding box for ground truth needs to be wider and the size needs to be constant to achieve better results. Figure 8 below shows the bounding box for ground truth for the original datasets, and that has been modified by standardization into $40 \times 18$ width and height which is empirically determined. As we can see, after standardization, the box is more consistent in size and includes more data to reduce over fitting.

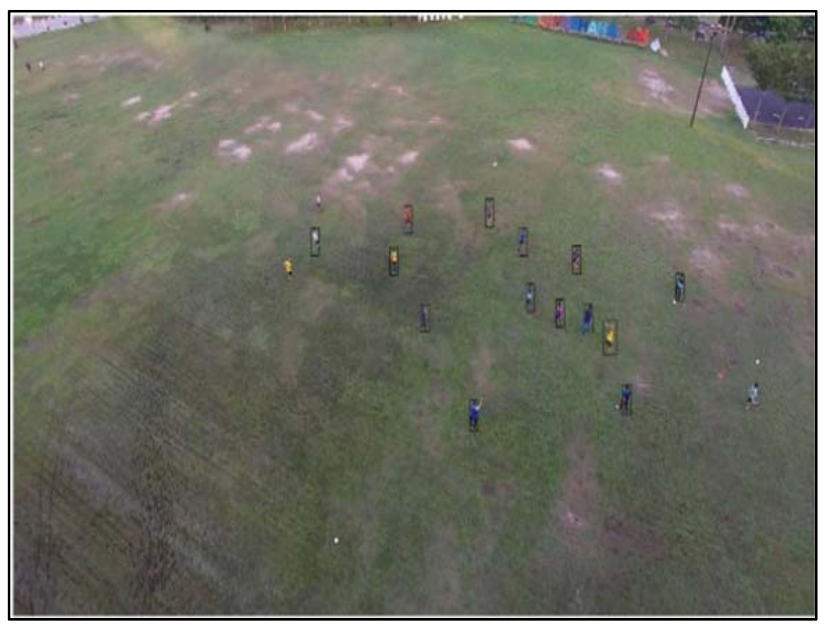

(a)

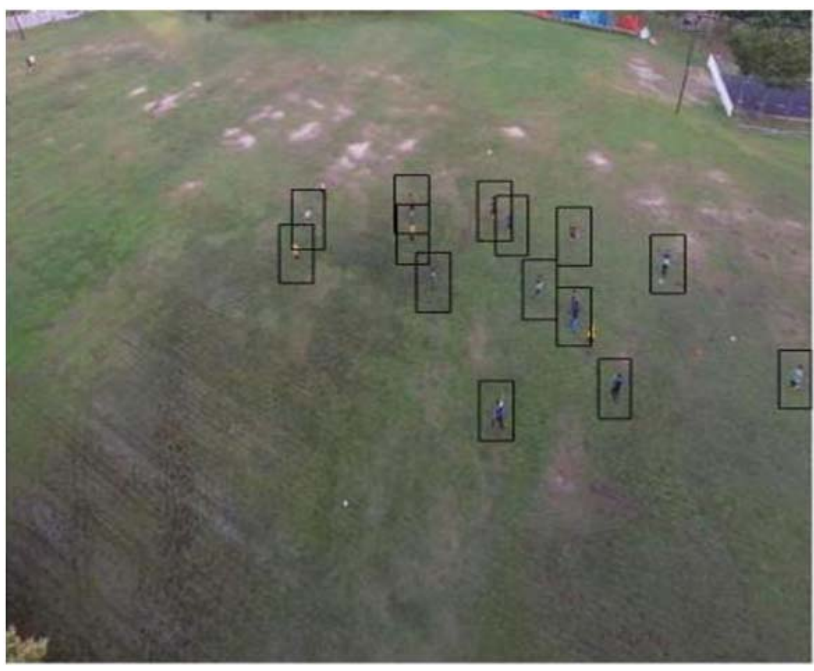

(b)

Figure 8. Several examples of bounding box (a) before bounding box standardization and (b) after bounding box standardization to $40 \times 18$

In order to test the detector, the resolution of the image dataset extracted from the video needs to be resized from $4096 \times 2160 p$ ( $4 K$ ) to $448 \times 448$ to suit the image input layer and to reduce the heavy computation overload as well. Table 4 below shows the result of YOLO MobileNet before and after through bounding box standardization.

Table 4. Test precision (AP) before and after bounding box standardization

\begin{tabular}{|c|c|c|c|c|}
\hline \multirow{2}{*}{$\begin{array}{c}\text { Training } \\
\text { Height }\end{array}$} & \multirow{2}{*}{$\begin{array}{c}\text { Testing } \\
\text { Height }\end{array}$} & \multicolumn{3}{|c|}{$\begin{array}{c}\text { Test Precision (AP) for YOLO } \\
\text { MobileNet }\end{array}$} \\
\cline { 2 - 5 } & & Before & After & Improvement \\
\hline \multirow{3}{*}{$\mathbf{1 0} \mathbf{~ m}$} & $10 \mathrm{~m}$ & 0.71 & 0.93 & 0.22 \\
\cline { 2 - 5 } & $20 \mathrm{~m}$ & 0.08 & 0.76 & 0.68 \\
\cline { 2 - 5 } & $30 \mathrm{~m}$ & 0.00 & 0.70 & $\mathbf{0 . 7}$ \\
\hline \multirow{3}{*}{$\mathbf{2 0} \mathbf{~ m}$} & $10 \mathrm{~m}$ & 0.35 & 0.88 & 0.53 \\
\cline { 2 - 5 } & $20 \mathrm{~m}$ & 0.71 & 0.82 & 0.11 \\
\cline { 2 - 5 } & $30 \mathrm{~m}$ & 0.45 & 0.91 & 0.46 \\
\hline \multirow{3}{*}{$\mathbf{3 0} \mathbf{~ m}$} & $10 \mathrm{~m}$ & 0.15 & 0.77 & 0.62 \\
\cline { 2 - 5 } & $20 \mathrm{~m}$ & 0.51 & 0.78 & 0.27 \\
\cline { 2 - 5 } & $30 \mathrm{~m}$ & 0.66 & 0.94 & 0.28 \\
\hline
\end{tabular}

As tabulated in Table 4, significant improvements are observed in the tested AP for every detector after bounding box standardization. For example, in the 10-meter testing on 10-meter training data, a significant increase is shown after bounding box standardization from 0.71 AP to 0.93 AP. Further at 20 meters and 30 meters tests on 10 meters training data, the AP increased significantly too from 0.08 AP to $0.76 \mathrm{AP}$ and 0.00 to $0.70 \mathrm{AP}$ after bounding box standardization. The largest improvement is by 0.7 AP for test on 30m height using 10-meter model.

Recall that for every detector, there are three types of height detector specifically 10-meter, 20-meter, and 30-meter detector. In addition, every detector is tested with three different heights that are 10 meter, 20 meter, and 30 meter which are captured at entirely different sessions. In addition, for every testing, the average precision and log-average miss rate are computed. However, log average precision is similar to average precision and referring to undetected objects. Recall that the YOLO detector is very sensitive besides height such as the resolution of the camera, ambient light, angle of the camera to the object with the camera. All these factors will affect detector performance. Fully connected layers increase the receptive field of each final activation to the full-image-size but will not affect network invariant to aspect-ratio. Table 6 shows the result for every detector that is used in this experiment that is ACF, YOLO MobileNet, and YOLO ResNet.

Based on the test result, the YOLO MobileNet precision was higher compared to the YOLO ResNet50 and ACF. The average precision (AP) for YOLO MobileNet, YOLO ResNet50, and ACF is $0.83,0.18$, and $0.46 \mathrm{AP}$ respectively. The AP for each training data for these three detectors is also tabulated in Table 5. The high value of AP means that the detector gets better precision and higher recall. Higher precision relates to a low false positive rate and higher recall relates to a low false-negative rate. 
Table 5. Precision result for ACF, YOLO MobileNet, and YOLO ResNet50

\begin{tabular}{|c|c|c|c|c|c|c|c|}
\hline \multirow[b]{2}{*}{$\begin{array}{c}\text { Training } \\
\text { Data }\end{array}$} & \multirow[b]{2}{*}{$\begin{array}{c}\text { Testing } \\
\text { Data }\end{array}$} & \multicolumn{3}{|c|}{ Test Precision (AP) } & \multicolumn{3}{|c|}{ Average Test AP for Each Training Data } \\
\hline & & $\begin{array}{c}\text { YOLO } \\
\text { MobileNet }\end{array}$ & $\begin{array}{c}\text { YOLO } \\
\text { ResNet50 }\end{array}$ & $\mathbf{A C F}$ & $\begin{array}{c}\text { YOLO } \\
\text { MobileNet }\end{array}$ & $\begin{array}{c}\text { YOLO } \\
\text { ResNet50 }\end{array}$ & $\mathbf{A C F}$ \\
\hline \multirow{3}{*}{$10 \mathrm{~m}$} & $10 \mathrm{~m}$ & 0.93 & 0.37 & 0.8 & \multirow{3}{*}{0.80} & \multirow{3}{*}{0.22} & \multirow{3}{*}{0.50} \\
\hline & $20 \mathrm{~m}$ & 0.76 & 0.16 & 0.5 & & & \\
\hline & $30 \mathrm{~m}$ & 0.70 & 0.13 & 0.2 & & & \\
\hline \multirow{3}{*}{$20 \mathrm{~m}$} & $10 \mathrm{~m}$ & 0.88 & 0.24 & 0.5 & \multirow{3}{*}{0.87} & \multirow{3}{*}{0.20} & \multirow{3}{*}{0.50} \\
\hline & $20 \mathrm{~m}$ & 0.82 & 0.26 & 0.6 & & & \\
\hline & $30 \mathrm{~m}$ & 0.91 & 0.11 & 0.4 & & & \\
\hline \multirow{3}{*}{$30 \mathrm{~m}$} & $10 \mathrm{~m}$ & 0.77 & 0.19 & 0.2 & \multirow{3}{*}{0.83} & \multirow{3}{*}{0.11} & \multirow{3}{*}{0.37} \\
\hline & $20 \mathrm{~m}$ & 0.78 & 0.08 & 0.3 & & & \\
\hline & $30 \mathrm{~m}$ & 0.94 & 0.05 & 0.6 & & & \\
\hline \multicolumn{2}{|c|}{ Average Test AP } & 0.83 & 0.18 & 0.46 & & & \\
\hline
\end{tabular}

Subsequently, the results attained are also plotted based on the Precision-Recall Curve for to better visualize and identify the most optimum detector based on each height and dataset used as well. These are shown in Figure 9 through Figure 11 with respect of each height evaluated.
Firstly, Figure 9 shows the test result for the 10, 20, and 30-meter test. It is observed that the precisionrecall curve for YOLO MobileNet is the best as compared to YOLO ResNet and ACF with a 10-meter test on 10-meter training data as the most optimum as depicted in Figure 8.

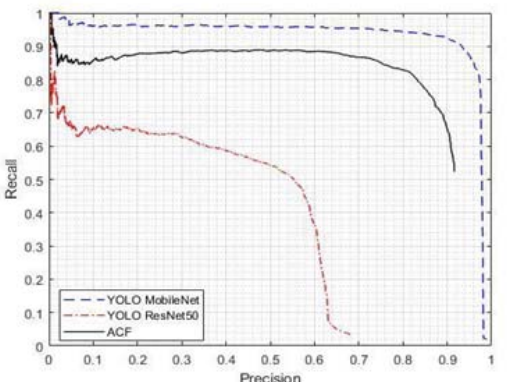

(a)

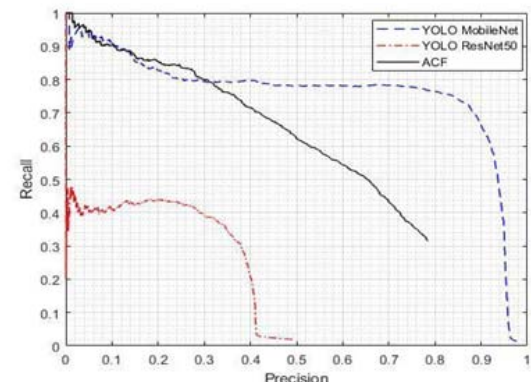

(b)

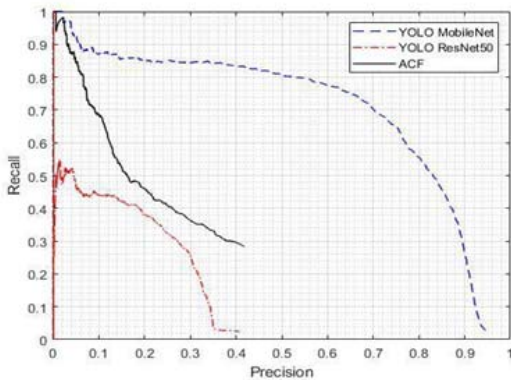

(c)

Figure 9. Testing result for (a) 10 meter, (b) 20 meter, and (c) 30 meter test data using 10-meter training data

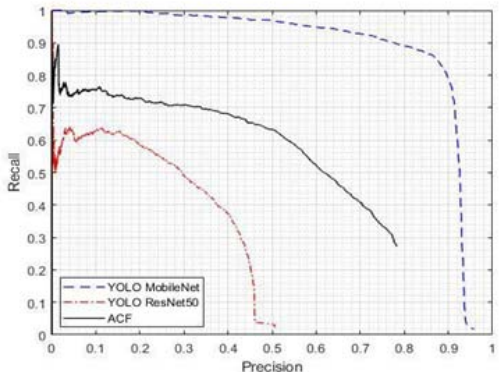

(a)

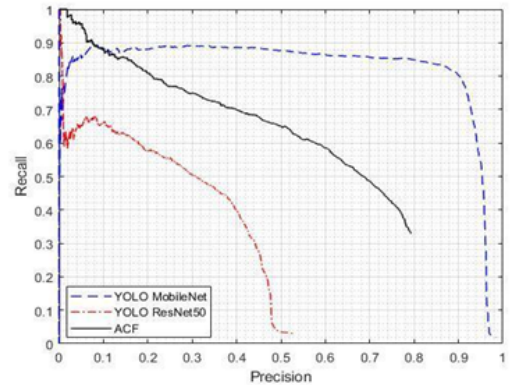

(b)

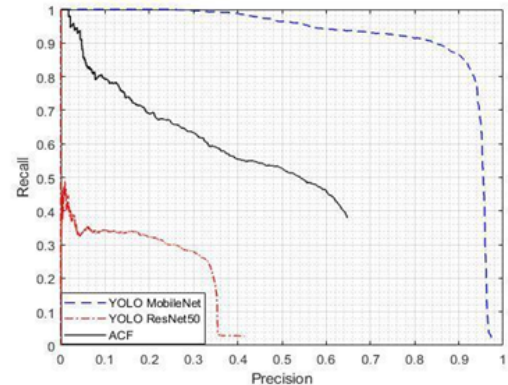

(c)

Figure 10. Testing result for (a) 10 meter, (b) 20 meter, and (c) 30 meter test data using 20-meter training data

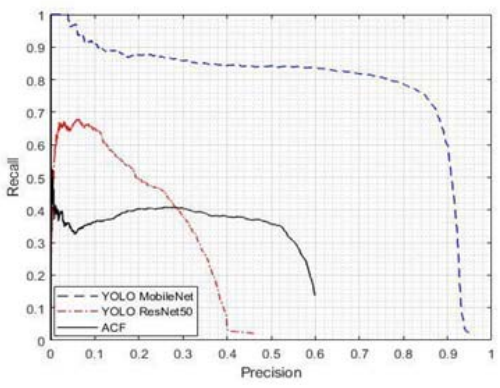

(a)

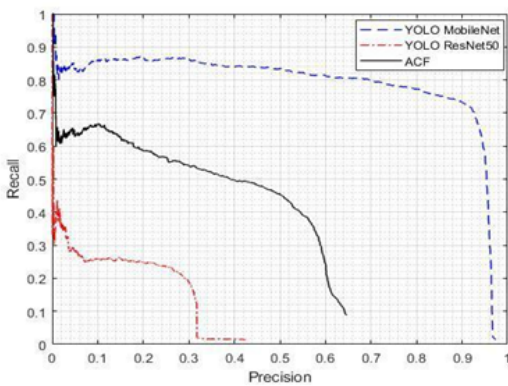

(b)

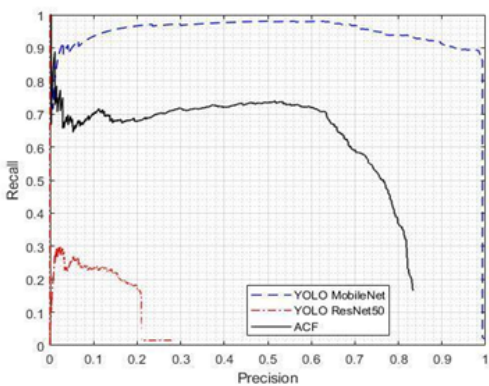

(c)

Figure 11. Testing result for (a) 10 meter, (b) 20 meter, and (c) 30 meter test data using 30-meter training data 
Next, Figure 10 shows the test results for 10meter, 20-meter, and 30-meter test. Once again it is observed that YOLO MobileNet is the best amongst the three detectors. YOLO MobileNet detector still has a better curve with $0.88 \mathrm{AP}(10 \mathrm{~m}), 0.82 \mathrm{AP}(20 \mathrm{~m})$, and $0.91 \mathrm{AP}(30 \mathrm{~m})$. Surprisingly, the YOLO MobileNet in Figure 9 (a) and 9 (b) get higher for the area under the curve (AUC) for 20-meter training data compared to Figure 9 (b).

Finally, Figure 11 depicts the testing result for 10meter, 20-meter and 30-meter testing using 30-meter training data. As mentioned earlier, the size of human forms is very small at this particular height. Based on the plots, YOLO MobileNet yields the best results among all.

\section{Conclusion}

In conclusion, three type of detector that is YOLO MobileNet, YOLO ResNet50 and ACF are used to evaluate and validate the effectiveness of their functionally as a detector using datasets from aerial images taken from different heights. The three heights identified are $10 \mathrm{~m}, 20 \mathrm{~m}$, and $30 \mathrm{~m}$ respectively, and results attained showed that the height of datasets adversely affects the detection result. Conversely, during dataset acquisition, the inconsistent size of humans in the images also occurred since at a higher location, the human form images will be smaller. Upon completion of dataset acquisition, the efficiency and precision of the detectors are enhanced in determining the highest test precision (AP) and this is achieved by introducing the bounding box standardization. Further, the average test precision result for YOLO MobileNet was $0.87 \mathrm{AP}$ using $20-\mathrm{m}$ training data which is the highest as compared to YOLO ResNet50 and ACF for all three training datasets used. Besides that, it was also identified from this study that several other factors that affected the training process of detector include resolution of video during collecting datasets process, the ambient and the angle of the camera to the object, and these are the parameters to be investigated in our future work.

\section{Acknowledgements}

The authors would like to thank the Ministry of Education (MOE) Malaysia for providing the grant 600IRMI/TRGS 5/3 (001/2019)-1, and the Research Management Centre (RMC) of Universiti Teknologi MARA for supporting this research work.

\section{References}

[1]. Waharte, S., \& Trigoni, N. (2010, September). Supporting search and rescue operations with UAVs. In 2010 International Conference on Emerging Security Technologies (pp. 142-147). IEEE.

[2]. Yim, J., Park, H., Kwon, E., Kim, S., \& Lee, Y. T. (2018, January). Low-power image stitching management for reducing power consumption of UAVs for disaster management system. In 2018 IEEE International Conference on Consumer Electronics (ICCE) (pp. 1-3). IEEE.

[3]. AlDahoul, N., Sabri, A. Q. M., \& Mansoor, A. M. (2018). Real-Time Human Detection for Aerial Captured Video Sequences via Deep Models. Computational Intelligence and Neuroscience, 2018, 1-14.

[4]. Aung, N. L., \& Koldaev, V. D. (2018, September). The Algorithm of Stitching Aerial Images Frames in Video Surveillance Systems. In 2018 International Russian Automation Conference (RusAutoCon) (pp. 15). IEEE.

[5]. Wang, X., Han, T. X., \& Yan, S. (2009, September). An HOG-LBP human detector with partial occlusion handling. In 2009 IEEE 12th international conference on computer vision (pp. 32-39). IEEE.

[6]. Lowe, D. G. (2004). Distinctive image features from scale-invariant keypoints. International Journal of Computer Vision, 60(2), 91-110.

[7]. Dalal, N., \& Triggs, B. (2005, June). Histograms of oriented gradients for human detection. In 2005 IEEE computer society conference on computer vision and pattern recognition (CVPR'05) (Vol. 1, pp. 886-893). IEEE.

[8]. Krizhevsky, A., Sutskever, I., \& Hinton, G. E. (2012). Imagenet classification with deep convolutional neural networks. Advances in neural information processing systems, 25, 1097-1105.

[9]. Szegedy, C., Liu, W., Jia, Y., Sermanet, P., Reed, S., Anguelov, D., ... \& Rabinovich, A. (2015). Going deeper with convolutions. In Proceedings of the IEEE conference on computer vision and pattern recognition (pp. 1-9).

[10]. Taigman, Y., Yang, M., Ranzato, M. A., \& Wolf, L. (2014). Deepface: Closing the gap to human-level performance in face verification. In Proceedings of the IEEE conference on computer vision and pattern recognition (pp. 1701-1708).

[11]. Ciregan, D., Meier, U., \& Schmidhuber, J. (2012, June). Multi-column deep neural networks for image classification. In 2012 IEEE conference on computer vision and pattern recognition (pp. 3642-3649). IEEE.

[12]. LeCun, Y., Jackel, L. D., Bottou, L., Cortes, C., Denker, J. S., Drucker, H., ... \& Vapnik, V. (1995). Learning algorithms for classification: A comparison on handwritten digit recognition. Neural networks: the statistical mechanics perspective, 261(276), 2. 
[13]. Toshev, A., \& Szegedy, C. (2014). Deeppose: Human pose estimation via deep neural networks. In Proceedings of the IEEE conference on computer vision and pattern recognition (pp. 1653-1660).

[14]. Girshick, R., Donahue, J., Darrell, T., \& Malik, J. (2014). Rich feature hierarchies for accurate object detection and semantic segmentation. In Proceedings of the IEEE conference on computer vision and pattern recognition (pp. 580-587).

[15]. Sermanet, P., Kavukcuoglu, K., Chintala, S., \& LeCun, Y. (2013). Pedestrian detection with unsupervised multi-stage feature learning. In Proceedings of the IEEE conference on computer vision and pattern recognition (pp. 3626-3633).

[16]. Babenko, A., Slesarev, A., Chigorin, A., \& Lempitsky, V. (2014, September). Neural codes for image retrieval. In European conference on computer vision (pp. 584-599). Springer, Cham.

[17]. Wan, J., Wang, D., Hoi, S. C. H., Wu, P., Zhu, J., Zhang, Y., \& Li, J. (2014, November). Deep learning for content-based image retrieval: A comprehensive study. In Proceedings of the 22nd ACM international conference on Multimedia (pp. 157-166).

[18]. Botalb, A., Moinuddin, M., Al-Saggaf, U. M., \& Ali, S. S. (2018, August). Contrasting convolutional neural network (CNN) with multi-layer perceptron (MLP) for big data analysis. In 2018 International conference on intelligent and advanced system (ICIAS) (pp. 1-5). IEEE.

[19]. Vo, D. M., \& Le, T. H. (2016, September). Deep generic features and SVM for facial expression recognition. In 2016 3rd National Foundation for Science and Technology Development Conference on Information and Computer Science (NICS) (pp. 8084). IEEE.

[20]. He, K., Zhang, X., Ren, S., \& Sun, J. (2015). Spatial pyramid pooling in deep convolutional networks for visual recognition. IEEE transactions on pattern analysis and machine intelligence, 37(9), 1904-1916.
[21]. Girshick, R. (2015). Fast r-cnn. In Proceedings of the IEEE international conference on computer vision (pp. 1440-1448).

[22]. Ren, S., He, K., Girshick, R., \& Sun, J. (2017). Faster R-CNN: Towards Real-Time Object Detection with Region Proposal Networks. IEEE Transactions on Pattern Analysis and Machine Intelligence, 39(6), 1137-1149.

[23]. Liu, W., Anguelov, D., Erhan, D., Szegedy, C., Reed, S., Fu, C. Y., \& Berg, A. C. (2016, October). Ssd: Single shot multibox detector. In European conference on computer vision (pp. 21-37). Springer, Cham.

[24]. Redmon, J., Divvala, S., Girshick, R., \& Farhadi, A. (2016). You only look once: Unified, real-time object detection. In Proceedings of the IEEE conference on computer vision and pattern recognition (pp. 779788).

[25]. Redmon, J., \& Farhadi, A. (2017). YOLO9000: better, faster, stronger. In Proceedings of the IEEE conference on computer vision and pattern recognition (pp. 7263-7271).

[26]. Jeong, H. J., Park, K. S., \& Ha, Y. G. (2018, January). Image preprocessing for efficient training of YOLO deep learning networks. In 2018 IEEE International Conference on Big Data and Smart Computing (BigComp) (pp. 635-637). IEEE.

[27]. Howard, A. G., Zhu, M., Chen, B., Kalenichenko, D., Wang, W., Weyand, T., ... \& Adam, H. (2017). Mobilenets: Efficient convolutional neural networks for mobile vision applications. arXiv preprint arXiv:1704.04861.

[28]. Sandler, M., Howard, A., Zhu, M., Zhmoginov, A., \& Chen, L. C. (2018). Mobilenetv2: Inverted residuals and linear bottlenecks. In Proceedings of the IEEE conference on computer vision and pattern recognition (pp. 4510-4520). 